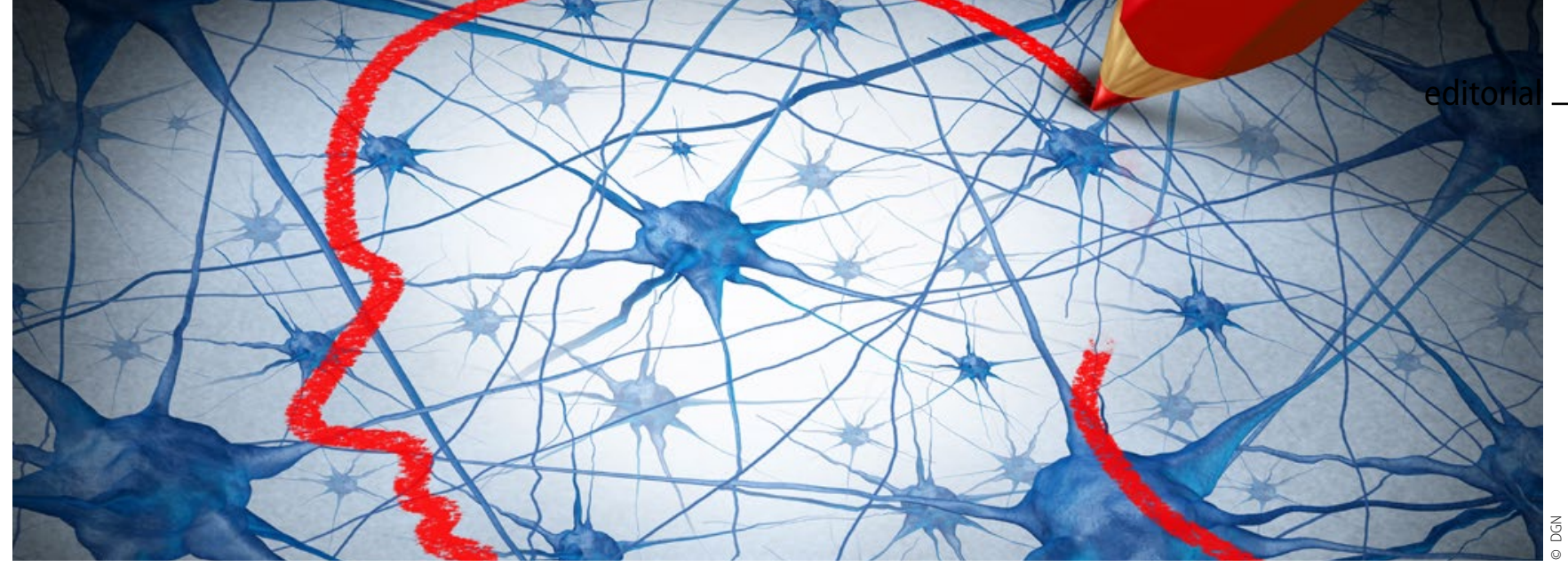

\title{
Wir sind Neurologie. - Eine Kampagne für die Schlüsselmedizin des 21. Jahrhunderts
}

Nie war es spannender, Neurologe zu sein: fantastische Möglichkeiten in der Diagnostik, kombiniert mit einer Vielfalt effektiver Therapien, sei es gegen Schlaganfall, Multiple Sklerose oder Morbus Parkinson. Bei vielen neurologischen Erkrankungen ist es uns gelungen, sowohl die Morbidität als auch die Mortalität zu reduzieren. Auf diese Errungenschaften können wir stolz sein. Die Neurologie ist die Schlüsselmedizin des 21. Jahrhunderts - denn Fortschritte in der Neurologie sind Fortschritte für die gesamte Gesellschaft.

\section{Erfolge sichtbar machen}

Um die Erfolge der Neurologie sichtbar zu machen, aber auch um die Ziele zu benennen, die noch vor uns liegen, startet die Deutsche Gesellschaft für Neurologie (DGN) die Imagekampagne „Wir sind Neurologie.“ Zum Auftakt geben 20 Botschafter aus unterschiedlichen Bereichen unseres Fachs - Ordinarien, Forscher, Klinikdirektoren, Oberärzte, Assistenzärzte, niedergelassene Neurologen ebenso wie Medizinstudierende - zentralen neurologischen Themen eine Stimme.

„Ist es wirklich nötig, dass wir die DGN mit einer Öffentlichkeitskampagne weiter stärken“, wurde ich in den vergangenen Wochen schon mehrfach gefragt. "Ja“, davon sind wir, das Präsidium der DGN, überzeugt. Die Kampagne „Wir sind Neurologie.“ wird in mehreren Phasen über die kommenden Jahre die Lobbyarbeit für die Neurologie stärken. Sie zeigt die Vielfalt unseres Fachs und ist gleichzeitig ein klares Bekenntnis zur Einheit der Neurologie. Sie schafft ein gemeinsames Bild und eine gemeinsame Vision von der Neurologie für ein starkes Wir-Gefühl. Die Stärkung unserer Fachgesellschaft wird in viele wichtige gesellschaftliche Bereiche ausstrahlen: in die Gesundheitswirtschaft genauso wie in die Universitäten, zur frühen Rekrutierung des Nachwuchses, in die Patientenschaft wie auch in die Politik.

In der älter werdenden Gesellschaft mit zunehmend knappen Ressourcen im Gesundheitssystem wird es künftig stärker darum gehen, wie Gelder verteilt werden, sowohl in der Forschung als auch in der Patientenversorgung. Als Neurologen müssen wir uns einerseits dafür stark machen, dass die neurologische Forschung intensiviert und die zunehmende Zahl der Menschen mit altersbezogenen neurologischen Erkrankungen gut versorgt wird. Gleichzeitig dürfen wir auch die jüngeren Patienten mit

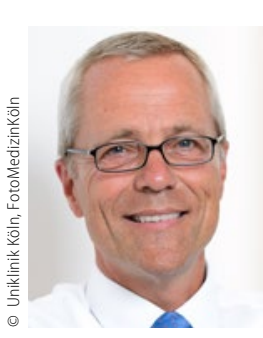

\author{
Prof. Dr. med. Gereon R. Fink \\ Präsident der Deutschen Gesellschaft \\ für Neurologie (DGN) \\ Direktor der Klinik und Poliklinik \\ für Neurologie, Universitätsklinik Köln \\ Kerpener Straße 62, 50937 Köln \\ E-Mail: fink@dgn.org
}

neurologischen Erkrankungen wie Multiple Sklerose nicht aus den Augen verlieren. Um dieser Verantwortung gerecht zu werden, müssen wir die Bedeutung der Neurologie in der medizinischen Versorgung stärker in den Fokus von Öffentlichkeit und Politik rücken.

\section{Talente binden}

Wir stehen jedoch nicht nur in einem Wettbewerb bei der Verteilung der finanziellen Ressourcen. Wir stehen auch in einem Wettbewerb um die Talente. Mit der Kampagne „Wir sind Neurologie." wollen wir gerade auch junge Menschen für das Fach begeistern. Die Botschaft ist klar: Jetzt ist die beste Zeit, um Neurologe zu sein. Das Fachgebiet hat einen großen Wandel durchgemacht, zu einem Fach mit nicht nur hervorragenden diagnostischen, sondern auch mit vielen neuen therapeutischen Möglichkeiten. Wir haben in den letzten fünf bis zehn Jahren in einigen Bereichen fantastische Erfolge gesehen. In anderen stehen wir weiterhin vor großen Herausforderungen, etwa beim Morbus Alzheimer oder bei Hirntumoren. Nur wenn wir es schaffen, junge, talentierte Köpfe für das Fach zu gewinnen, wird es uns mittel- und langfristig gelingen, wirksame Therapien gegen diese Krankheiten zu entwickeln und diese flächendeckend anbieten zu können. Hier hat die DGN mit den „Jungen Neurologen“ in den vergangenen Jahren schon viel erreicht. Zum Wohl unseres Fachs und im Sinne unserer Patienten dürfen wir uns damit aber noch lange nicht zufriedengeben.

Gereon R. Fink

Alle Informationen zur Kampagne finden Sie im Internet unter www.wir-sind-neurologie.de 